\title{
AN EXTENSION OF THE CONCEPT OF THE ORDER DUAL OF A RIESZ SPACE
}

\author{
W. A. J. LUXEMBURG AND J. J. MASTERSON
}

Introduction. Let $L$ be a $\sigma$-Dedekind complete Riesz space. In (8), H. Nakano uses an extension of the multiplication operator on a Riesz space into itself (analagous to the closed operator on a Hilbert space) to obtain a representation space for the Riesz space $L$. He calls such an operator a "dilatator operator on L." More specifically, he shows that the set of all dilatator operators $\mathfrak{D}(L)$, when suitable operations are defined, is a Dedekind complete Riesz space which is isomorphic to the space of all functions defined and continuous on an open dense subset of some fixed totally disconnected Hausdorff space. The embedding of $L$ in the function space is then obtained by showing that $L$ is isomorphic to a Riesz subspace of $\mathfrak{D}(L)$. Moreover, when $L$ is Dedekind complete, it is an ideal in $\mathfrak{D}(L)$, and the topological space is extremally disconnected.

Let $L$ now be an arbitrary Riesz space. We shall call a linear functional $\phi$ on $L$ a normal integral whenever $0 \leqslant u_{\tau} \downarrow 0$ in $L$ implies inf $|\phi(u)|=0$. (The terminology of Luxemburg and Zaanen in (4) will be adopted and knowledge of their series of notes on Banach Function Spaces, specifically $(\mathbf{4}, \mathbf{5}, \mathbf{6}, \mathbf{7})$, will be assumed.) $L_{n} \sim$, the space of all such linear functionals, was introduced by H. Nakano (cf., (8)) and has been studied more recently by S. Kaplan $(\mathbf{1}, \mathbf{2}, \mathbf{3})$ and, as mentioned above, by W. A. J. Luxemburg and A. C. Zaanen. The results of the last-mentioned authors will be used extensively.

It is the purpose of this paper to examine an extension of the normal integral on the Riesz space $L$, analogous to the dilatator operator of Nakano. The result of this extension is a Riesz space $\Gamma(L)$ of linear functionals defined on dense ideals of $L$, which has a number of the properties of the dual space $L_{n} \sim$. In fact, when $L_{n}{ }^{\sim}$ is separating on $L$, it can be embedded in $\Gamma(L)$ as a dense ideal.

Under weaker separation conditions (namely that $\Gamma(L)$ be separating on $L$ ) it will be shown that $\Gamma(L)$ is a universally complete lattice-ordered algebra which is isomorphic to Nakano's representation space $\mathfrak{D}(L)$. We can then obtain a representation of the Dedekind and universal completions of a certain class of Archimedean Riesz spaces. In doing so, we generalize the results contained in (6) concerning the Dedekind completion of $L$.

Received December 10, 1965, and in revised form, August 23, 1966. Work on this paper was supported in part by National Science Foundation Grant NSF-PG 6111 (to W. A. J. L.) and Grant NSF-PG-5973 (to J. J. M.). 
1. The Riesz space $\Gamma(L)$. We shall first recall the following terminology and results of the general theory of Riesz spaces.

Let $L$ be a Riesz space. An ideal $I \subset L$ is called order dense whenever for every $0 \leqslant u \in L$ there is a directed system $0 \leqslant u_{\tau} \uparrow\left(u_{\tau_{1}}, u_{\tau_{2}} \Rightarrow\right.$ there is a $u_{\tau_{3}}$ such that $u_{\tau_{3}} \geqslant u_{\tau_{1}}$ and $u_{\tau_{3}} \geqslant u_{\tau_{2}}$ ) of elements of $I$ such that $u=\sup u_{\tau}$, or equivalently, the smallest band containing $I$ is $L$.

In Archimedean Riesz spaces the following fundamental results hold: An ideal $I \subset L$ is order dense if and only if for every $0 \neq f \in L$ there is an element $g \in I$ such that $0 \neq|g| \leqslant|f|$. (5, Theorems 29.5 and 29.10). For every ideal $I \subset L$, the ideal $I \oplus I^{p}$ is order dense in $L$, where

$$
I^{p}=\{f: f \in L \text { and } \inf (|f|,|g|)=0 \text { for all } g \in I\} .
$$

If $L$ is Archimedean, then every normal integral is order bounded and its positive part and negative part are also normal integrals. For every ideal $I \subset L$, where $L$ is an Archimedean Riesz space, we shall denote by $I_{n}^{\sim}$ the Dedekind complete Riesz space of all normal integrals on $I$.

From now on we shall always assume that the Riesz space $L$ is Archimedean.

By $\mathfrak{\Im}$ we shall denote the family of all order dense ideals contained in $L$. Then $\mathfrak{\Im}$ is a filter basis. Let $\Phi=\bigcup\left\{I_{n} \sim: I \in \Im\right\}$. If $\phi \in \Phi$, we shall denote by $I_{\phi}$ its domain of definition. Thus $I_{\phi} \in \Im$ and $\phi \in\left(I_{\phi}\right)_{n} \sim$ for all $\phi \in \Phi$.

We then define the following relation on $\Phi: \phi_{1} \equiv \Im \phi_{2}$ whenever

$$
\left\{f: \phi_{1}(f)=\phi_{2}(f)\right\}
$$

contains an order dense ideal. Since $\Im$ is a filter basis, it follows immediately that the relation $\equiv \Im$ is an equivalence relation. The set of classes of equivalent elements will be denoted by $\Gamma(L)$. The elements of $\Gamma(L)$ will be temporarily denoted by $[\phi]$.

We can endow $\Gamma(L)$ with a Riesz space structure. To this end, for all real $a$ and all $[\phi] \in \Gamma(L)$ we set $a[\phi]=[a \phi]$. Furthermore, addition is being defined as follows: $\left[\phi_{1}\right]+\left[\phi_{2}\right]=\left[\phi_{3}\right]$ whenever there exist $\phi^{\prime}{ }_{1} \in\left[\phi_{1}\right]$, $\boldsymbol{\phi}_{2}{ }_{2} \in\left[\boldsymbol{\phi}_{2}\right]$, and $\boldsymbol{\phi}^{\prime}{ }_{3} \in\left[\phi_{2}\right]$ such that $\left\{f: \boldsymbol{\phi}_{1}^{\prime}(f)+\boldsymbol{\phi}_{2}^{\prime}(f)=\boldsymbol{\phi}^{\prime}{ }_{3}(f)\right\}$ contains an order dense ideal. Since $\Im$ is a filter basis, this definition does not depend on the particular representatives $\phi_{1}^{\prime}{ }_{1}, \phi_{2}^{\prime}$, and $\phi^{\prime}{ }_{3}$. It is now easy to see that with these definitions, $\Gamma(L)$ is a linear space over the real field $R$.

We shall now define $[\phi] \geqslant 0$ whenever there exists $\phi^{\prime} \in[\phi]$ such that $\left\{x: \phi^{\prime}(x) \geqslant 0\right\} \in \Im$. Again if $[\phi] \geqslant 0$, then $\left\{x: \phi^{\prime}(x)>0\right\} \in \Im$ for all $\phi^{\prime} \in[\phi]$. It is then easy to see that the set of all non-negative elements forms a cone in $\Gamma(L)$. We then set $\left[\phi_{1}\right] \leqslant\left[\phi_{2}\right]$ whenever $\left[\phi_{2}\right]-\left[\phi_{1}\right]=\left[\phi_{2}-\phi_{1}\right] \geqslant 0$. This orders $\Gamma(L)$ and the order structure is compatible with the linear space structure of $\Gamma(L)$. Since for every $[\phi] \in \Gamma(L)$ we have $[\phi] \leqslant\left[\phi^{+}\right]$, and if $[\psi] \geqslant 0$ such that $[\phi] \leqslant[\psi]$ we have $\left[\phi^{+}\right] \leqslant[\psi]$, it follows that $[\phi]^{+}$exists and is equal to $\left[\phi^{+}\right]$. Thus $\Gamma(L)$ is a Riesz space.

The special nature of the elements of $\Phi$ will enable us to show that $\Gamma(L)$ has the lifting property. To this end, we shall first establish a criterion for 
equivalence in $\Phi$. We may restrict the discussion to positive linear functionals since we have the obvious result:

$$
\phi_{1} \equiv \Im \phi_{2} \quad \text { if and only if } \phi_{1}{ }^{+} \equiv \Im \phi_{1}{ }^{+} \equiv \Im \phi_{2}{ }^{+} \text {and } \phi_{1}{ }^{-} \equiv \phi_{2}{ }^{-} \text {. }
$$

Let $0 \leqslant \phi \in \Phi$ and let $I_{\phi}$ be the order dense ideal on which it is defined. Then following section 30 in (5) we set for all $0 \leqslant u \in L$

$$
\bar{\phi}(u)=\sup \left(\phi(v): 0 \leqslant v \leqslant u \text { and } v \in I_{\phi}\right) .
$$

It is easy to see that $\bar{\phi}\left(u_{1}+u_{2}\right)=\bar{\phi}\left(u_{1}\right)+\bar{\phi}\left(u_{2}\right), \bar{\phi}\left(a u_{1}\right)=a \bar{\phi}\left(u_{1}\right)$ for all $0 \leqslant u_{1}, u_{2} \in L$ and $0 \leqslant a \in R$. Obviously we have $\bar{\phi}(u) \leqslant \bar{\phi}(v)$ whenever $0 \leqslant u \leqslant v \in L$. Thus $\bar{\phi}$ is the minimal additive and monotonic extension of $\phi$ to the positive cone $L^{+}$of $L$ with values in the extended real number system. For every $\phi \in \Phi$ we shall write $D_{\phi}=\{f: f \in L$ and $|\bar{\phi}|(|f|)<\infty\}$. Then $D_{\phi}$ is an order dense ideal containing $I_{\phi}$ and in fact the largest order dense ideal on which $|\phi|$ can be extended finitely. We shall now prove the following theorem.

Theorem 1.1. If $0 \leqslant \phi, \psi \in \Phi$, then $\phi \equiv \psi$ if and only if $\bar{\phi}=\bar{\psi}$ on $L^{+}$.

Proof. If $\bar{\phi}=\bar{\psi}$, then obviously $\phi \equiv \Im \psi$. Conversely, if $\phi \equiv \Im \psi$, then $\phi=\psi$ on some order dense ideal $I \subset I_{\phi} \cap I_{\psi}$. Since $\phi$ as well as $\psi$ is normal, we obtain $\phi(u)=\sup \left(\phi(v): 0 \leqslant v \leqslant u\right.$ and $\left.v \in I_{\phi}\right)$ for all $0 \leqslant u \in I_{\phi}$ and similarly for $\psi$, and so for all $0 \leqslant u \in L$ we have

$$
\bar{\phi}(u)=\sup (\phi(v): 0 \leqslant v \leqslant u \text { and } v \in I)
$$

and similarly for $\psi$. Hence $\bar{\phi}=\bar{\psi}$.

Theorem 1.2. For every $0 \leqslant \phi \in \Phi$ we have that if $0 \leqslant u_{\tau} \uparrow u$ in $L$, then $\phi(u)=\sup \phi\left(u_{\tau}\right)$.

Proof. Let $0 \leqslant v \leqslant u$ and $v \in I_{\phi}$. Then since $\phi$ is normal, we have $\phi(v)=\sup \phi\left(\inf \left(u_{\tau}, v\right)\right) \leqslant \sup \bar{\phi}\left(u_{\tau}\right)$. Hence, $\bar{\phi}(u)=\sup \bar{\phi}_{\tau}(u)$.

Corollary 1.3. For every $\phi \in \Phi$ we have that the restriction of $\bar{\phi}$ to $D_{\phi}$ is normal.

This shows that for any $[\phi] \in \Gamma(L)$ there exists an element $\phi_{0} \in[\phi]$ such that for any $\phi^{\prime} \in[\phi]$ we have $I_{\phi^{\prime}} \subset I_{\phi_{0}}$ and $\phi^{\prime}=\phi_{0}$ on $I_{\phi^{\prime}}$. We shall identify $\Gamma(L)$ with the set of its maximal elements. If we denote temporarily by $\phi$ the maximum element determined by $[\phi] \in \Gamma(L)$, then we have obviously $\left[\phi_{3}\right]=\left[\phi_{1}\right]+\left[\phi_{2}\right]$ if and only if $\phi_{3}^{\wedge}=\phi_{1}^{\wedge}+\phi_{2}^{\wedge}$ and $\dot{D}_{\phi_{3}}=D_{\phi_{1}} \wedge \cap{D_{\phi_{2}} \wedge}_{\text {; }}$; and $\left[\phi_{1}\right] \leqslant\left[\phi_{2}\right]$ if and only if $\phi_{1}^{\wedge} \leqslant \phi_{2}^{\wedge}$ and $D_{\phi_{2}} \wedge \subset D_{\phi_{1} \wedge}$, i.e., the set of maximal elements forms a set of representatives of $\Gamma(L)$ of its Riesz space structure.

In place of $[\phi]$ we shall denote the elements of $\Gamma(L)$ by $\phi$ and their domain of definition by $D_{\phi}$.

Let $L$ be an Archimedean Riesz space. If $\phi \in \Gamma(L)$ then we write $N_{\phi}=\left\{f: f \in D_{\phi}\right.$ and $\left.|\phi|(|f|)=0\right\}$ and $C_{\phi}=\left\{f: f \in D_{\phi}\right.$ and $\left.f \perp N_{\phi}\right\}$. Then

$$
N_{\phi} \oplus C_{\phi}
$$


is order dense in $D_{\phi}$. Observe that from Theorem 1.2 it follows that if $N_{|\bar{\phi}|}=\{f:|\bar{\phi}|(|f|)=0$ and $f \in L\}$, then $N_{|\bar{\phi}|}=N_{\phi}$, and so $N_{\phi}$ is a band in $L$ contained in $D_{\phi}$. Furthermore, $C_{\phi}$ is order dense in $N_{\phi}{ }^{P}=C_{\bar{\phi}}$.

From the fact that $L$ is Archimedean it follows that $\phi \neq 0$ if and only if $C_{\phi} \neq\{0\}$, and for every $\phi,|\phi|$ is strictly positive on $C_{\phi}$.

THEOREM 1.4. If $\phi, \psi \in \Gamma(L)$, then $\phi \perp \psi$ if and only if $C_{\phi} \perp C_{\psi}$, or $C_{\phi} \subset N_{\psi}$, or $C_{\psi} \subset N_{\phi}$.

Proof. This follows immediately from (5, Theorem 31.2(ii)).

Remark. Since $N_{\phi}$ and $N_{\psi}$ are bands in $L$, we have more generally that $\phi \perp \psi$ if and only if $C_{\bar{\phi}} \perp C_{\bar{\psi}}$, or $C_{\bar{\phi}} \subset N_{\psi}$, or $C_{\bar{\psi}} \subset N_{\phi}$.

Theorem 1.5. If $L$ is Archimedean, then $\Gamma(L)$ is Dedekind complete and universally complete.

Proof. We shall first show that $\Gamma(L)$ is Dedekind complete. To this end, we shall assume that $0 \leqslant \phi_{\tau} \uparrow \leqslant \phi_{0}$. Then $\phi_{\tau} \leqslant \phi_{0}$ on $D_{\phi_{0}}$ for all $\tau$. Hence, using the fact that $\left(D_{\phi_{0}}\right)_{n} \sim$ is Dedekind complete, we obtain that there is an element $\phi \in\left(D_{\phi_{0}}\right)_{n} \sim$ such that $\sup \phi_{\tau}=\phi$ on $D_{\phi_{0}}$. Then the maximal element determined by $\phi$ is obviously the least upper bound of the system $\left\{\phi_{\tau}\right\}$.

In order to show that $\Gamma(L)$ is universally complete we assume that $\left\{\phi_{\tau}\right\}$ is an orthogonal family of non-negative elements of $\Gamma(L)$. From Theorem 1.4 it follows that the carriers $C_{\phi \tau}$ of the elements $\phi_{\tau}$ are mutually disjoint. Let $I$ be the smallest ideal containing the ideals $C_{\phi \tau}$. Then there is a unique normal integral on $I$ which coincides with $\phi_{\tau}$ on every $C_{\phi \tau}$. By defining it to be equal to zero on $I^{P}$ we obtain an element of $\left(I \oplus I^{P}\right)_{n}$, and since $I \oplus I^{P}$ is order dense, its unique maximal extension is the required least upper bound.

We conclude this section with the following theorem.

TheOREm 1.6. $L_{n} \sim$ can be embedded in a one-to-one way onto an ideal in $\Gamma(L)$.

Proof. We need only observe that whenever $\phi_{1}, \phi_{2} \in L_{n} \sim$, then $\phi_{1}=\phi_{2}$ if and only if $\left\{f:\left|\phi_{1}-\phi_{2}\right|(f)=0\right\}$ is order dense.

2. The embedding of $L$ in $\Gamma^{2}(L)$. The following theorem is essential. Let $L$ be an Archimedean Riesz space.

Theorem 2.1. If $0 \leqslant \phi \in \Gamma(L)$ and $\bar{\phi}(u)=\infty$ for some $0<u \in L$, then there is an element $\psi \in \Gamma(L)$ such that $0 \leqslant \psi \leqslant \phi$ and $0<\psi(u)<\infty$.

Proof. From $\bar{\phi}(u)=\infty$ it follows that there is an element $0<u_{0}<u$ such that $u_{0} \in C_{\phi}$. Since $L$ is Archimedean, there is an index $n_{0}$ such that $\left(n_{0} u_{0}-u\right)^{+}=v_{0}>0$. Observe that $v_{0} \in C_{\phi}$. We shall now show that $u_{k}=\inf \left(u, k v_{0}\right) \leqslant n_{0} u_{0}$ for all $k=1,2, \ldots$ To this end, observe that $\inf \left(u_{k},\left(n_{0} u_{0}-u\right)^{-}\right)=0$, and so $u-n_{0} u_{0}=\left(n_{0} u_{0}-u\right)^{-}-\left(n_{0} u_{0}-u\right)^{+}$ 
implies that $\inf \left(u_{k},\left(u-n_{0} u_{0}\right)\right) \leqslant 0$. Hence, $\inf \left(u_{k}+n_{0} u_{0}\right) \leqslant n_{0} u_{0}$. Since $u_{k} \leqslant u(k=1,2, \ldots)$ and $u_{k} \leqslant u_{k}+n_{0} u_{0}(k=1,2, \ldots)$ we obtain

$$
u_{k} \leqslant \inf \left(u_{k}+n_{0} u_{0}, u\right) \leqslant n_{0} u_{0} \quad \text { for all } k=1,2, \ldots
$$

Now we set $\psi(v)=\sup _{k} \phi\left(\inf \left(v, k v_{0}\right)\right)$ for all $0 \leqslant v \in L^{+}$. Then $\psi$ is additive and monotone on $L^{+}, \psi \leqslant \phi$ on $L^{+}, 0<\psi(u)<\infty$, and $\psi$ is finite on the order dense ideal $I_{v_{0}} \oplus I_{v_{0}}{ }^{P}$, where $I_{v_{0}}$ is the ideal generated by $v_{0}$. Hence the unique maximal extension of $\psi$ satisfies the conditions of the theorem.

We recall that a linear subspace $V$ of a Riesz space $L$ is called a Riesz subspace of $L$ whenever $f \in V$ implies $|f| \in V$ and, in that case, $\sup (f, g) \in V$ and $\inf (f, g) \in V$ for all $f, g \in V$. A Riesz subspace $V$ of $L$ is called order dense whenever, for every $0 \leqslant u \in L$, we have $u=\sup (v: 0 \leqslant v \leqslant u$ and $v \in V)$. Then (1, Theorem 59.1) states: A Riesz subspace $V$ of an Archimedean Riesz space $L$ is order dense in $L$ whenever, for every $0 \neq f \in L$, there is an element $g \in V$ such that $0 \neq|g| \leqslant|f|$.

Theorem 2.2. Any Archimedean Riesz space L can be embedded onto a Riesz subspace of $\Gamma^{2}(L)$.

Proof. Let $I_{u}=\left\{\phi: \phi \in \Gamma(L)\right.$ and $\left.u \in D_{\phi}\right\}$, where $0 \leqslant u \in L$. Then define $u^{\prime \prime}$ on $\Gamma(L)$ as follows: $u^{\prime \prime}(\phi)=\phi(u)$ for all $\phi \in I_{u}$. From Theorem 2.1 and the fact that $\Gamma(L)$ is Archimedean it follows that $I_{u}$ is an order dense ideal in $\Gamma(L)$. The fact that $u^{\prime \prime}$ is normal in $I_{u}$ is trivial. Furthermore, the embedding $u \rightarrow u^{\prime \prime}$ of $L$ into $\Gamma^{2}(L)$ preserves the Riesz space structure and so maps $L$ onto a Riesz subspace of $\Gamma^{2}(L)$.

In the following we shall always identify any Archimedean Riesz space with this Riesz subspace of $\Gamma^{2}(L)$, and so we shall write $L \subset \Gamma^{2}(L)$.

It is obvious that the mapping $u \rightarrow u^{\prime \prime}$ of $L$ into $\Gamma^{2}(L)$ is one-to-one if and only if $\Gamma(L)$ is separating on $L$, i.e., for every $0<u \in L$ there is an element $0<\phi \in \Gamma(L)$ such that $0<\phi(u)<\infty$. According to Theorem 2.1 this is equivalent to the statement that for every $0<u \in L$ there is an element $0<\phi \in \Gamma(L)$ such that $\phi(u)>0$.

When $\Gamma(L)$ is separating on $L$ we can prove a great deal more about the embedding. In order to simplify the discussion we shall briefly recall the notion of a Dedekind completion and a universal completion of an Archimedean Riesz space.

Let $L$ and $L^{\prime}$ be Riesz spaces.

The Riesz space $L^{\prime}$ is called a Dedekind completion of $L$ whenever:

(i) $L^{\prime}$ is Dedekind complete (every non-empty subset which is bounded above has a least upper bound).

(ii) There is a one-to-one mapping of $L$ onto a Riesz subspace of $L^{\prime}$ which preserves the Riesz space structure of $L$ (we shall always assume $L$ to be identified with this Riesz subspace). 
(iii) For every $f \in L^{\prime}$ we have

$$
f=\sup (g: g \in L \text { and } g \leqslant f)=\inf (h: h \in L \text { and } f \leqslant h) \text {. }
$$

It was shown by Nakano that a Riesz space has a Dedekind completion if and only if it is Archimedean, and in that case any two Dedekind completions are isomorphic. In general it is not so easy to describe the Dedekind completion of an Archimedean Riesz space $L$ in terms of $L$. In $(6, \S 32)$ a simple representation was given for the Dedekind completion of $L$ whenever $L_{n} \sim$ is separating. We shall now be able to extend this result to the case that $\Gamma(L)$ is separating.

The following definition is a slight generalization of a definition of Nakano.

The Riesz space $L^{\prime}$ is called a universal completion of $L$ whenever:

(ii) $\mathrm{L}^{\prime}$ is Dedekind complete and universally complete.

(ii) There is a one-to-one mapping of $L$ onto an order dense Riesz subspace of $L^{\prime}$ (we shall always identify $L$ with this Riesz subspace).

Analogously we have the result that a Riesz space admits a universal completion if and only if it is Archimedean and in that case any two universal completions are isomorphic. It follows immediately from (1, Theorem 59.1) that if $L^{\prime}$ is a universal completion of $L$, then the ideal in $L^{\prime}$ generated by $L$ is a Dedekind completion of $L$.

If $L$ is Dedekind complete, then this definition is equivalent to Nakano's definition of a universal completion of a Dedekind complete Riesz space.

In general the universal completion of an Archimedean Reisz space $L$ is not easy to describe. But in the case of $\Gamma(L)$ separating on $L$ we have the following theorem.

Theorem 2.3. If $\Gamma(L)$ is separating (and hence $L$ is Archimedean), then $L$ can be identified in a one-to-one manner with an order dense Riesz subspace of $\Gamma^{2}(L)$. Thus $\Gamma^{2}(L)$ is a universal completion of $L$ and the ideal in $\Gamma^{2}(L)$ generated by $L$ is a Dedekind completion of $L$.

Proof. We shall first show that whenever $0<u^{\prime \prime}<u$, where $u \in L$ and $u^{\prime \prime} \in \Gamma^{2}(L)$, then there exists an element $0<u_{0} \in L$ such that $u_{0}<u^{\prime \prime}$. From $u^{\prime \prime}<u$ and the fact that $\Gamma^{2}(L)$ is Archimedean it follows that for sufficiently small $0<\lambda \in R$ the element $u^{\prime \prime}{ }_{\lambda}=\left(u^{\prime \prime}-\lambda u\right)^{+}$satisfies $u^{\prime \prime}{ }_{\lambda}>0$. Let $C_{\lambda}$ denote the carrier of $u^{\prime \prime}{ }_{\lambda}$ in $\Gamma(L)$. Since $u^{\prime \prime}{ }_{\lambda}>0$, there is an element $0<\phi_{0} \in C_{\lambda}$ such that $u^{\prime \prime}{ }_{\lambda}\left(\phi_{0}\right)>0$, and so $u^{\prime \prime}<u$ implies that $\phi_{0}(u)>0$. Then there exists an element $0<v \in C_{\phi_{0}}$ such that $0<v \leqslant u, \phi_{0}(v)>0$, and $\psi(v)=0$ for all $\psi \in \Gamma(L)$ and $\psi \perp \phi_{0}$ (Theorem 1.4). Let $0 \leqslant \phi \in \Gamma(L)$ and let $\phi$ be in the domain $D^{\prime \prime}{ }_{\lambda}$ of $u^{\prime \prime}{ }_{\lambda}$. Since $\Gamma(L)$ is Dedekind complete, the order dense ideal $D^{\prime \prime}{ }_{\lambda}$ is a Riesz space in its own right and also Dedekind complete, and so $D^{\prime \prime}{ }_{\lambda}=C_{\lambda} \oplus N_{\lambda}$, where $N_{\lambda}$ is the null ideal of $u^{\prime \prime}{ }_{\lambda}$ in $\Gamma(L)$. Let $\phi_{\lambda}$ be the component of $\phi$ in $C_{\lambda}$. Then we have

$$
\left(u^{\prime \prime}-\lambda u\right)\left(\phi_{\lambda}\right)=\left(u^{\prime \prime}-\lambda u\right)^{+}\left(\phi_{\lambda}\right)>0,
$$

and so $u^{\prime \prime}\left(\phi_{\lambda}\right) \geqslant \lambda \phi_{\lambda}(u)$. From the latter inequality we conclude that

$$
u^{\prime \prime}(\phi) \geqslant u^{\prime \prime}\left(\phi_{\lambda}\right) \geqslant \lambda \phi_{\lambda}(v)=\phi(\lambda v)
$$


where the last equality follows from the fact that $\phi-\phi_{\lambda} \perp \phi_{0}$ and the definition of $v$. Hence, $u^{\prime \prime}(\phi)>\phi(\lambda v)$ for all $0 \leqslant \phi$ in the order dense ideal $D^{\prime \prime}{ }_{\lambda}$ of $\Gamma(L)$, and this implies that $u^{\prime \prime}>\lambda v=u_{0}$ in $\Gamma^{2}(L)$.

In order to complete the proof we have to show that if $0<u^{\prime \prime} \in \Gamma^{2}(L)$ and $\inf \left(u^{\prime \prime}, u\right)=0$ for all $0 \leqslant u \in L$, then $u^{\prime \prime}=0$. Let $I$ be the ideal in $\Gamma(L)$ which is generated by the family of all carriers in $\Gamma(L)$ of the elements of $L$. Then it is easy to see that $I$ is order dense in $\Gamma(L)$. From inf $\left(u^{\prime \prime}, u\right)=0$ for all $0<u \in L$ it follows from Theorem 1.4 that the carrier $C_{u^{\prime \prime}}$ of $u^{\prime \prime}$ in $\Gamma(L)$ satisfies $C_{u^{\prime \prime}} \subset I^{P}=\{0\}$, and so $u^{\prime \prime}=0$. This completes the proof of the theorem.

Corollary 2.4. If $\Gamma(L)$ is separating, then $L$ is Dedekind complete if and only if $L$ is an ideal in $\Gamma^{2}(L)$, and hence $L$ is an order dense ideal in $\Gamma^{2}(L)$.

The following theorem establishes a condition for $\Gamma(L)$ to be separating.

THEOREM 2.5. $\Gamma(L)$ is separating if and only if there exists an order dense ideal $I \subset L$ such that $I_{n} \sim$ is separating on $I$, and in that case there exists an order dense ideal which admits a strictly positive normal integral.

Proof. Assume first that $I_{n} \sim$ is separating for some order dense ideal $I$ in $L$. Let $0<u \in L$. Then there is an element $v \in I$ such that $0<v \leqslant u$. Now let $0 \leqslant \phi \in I_{n} \sim$ be such that $\phi(v)>0$. If $\bar{\phi}(u)<\infty$, then the proof is finished. If $\bar{\phi}(u)=\infty$, then according to Theorem 2.1 there is an element $0<\psi \leqslant \phi$, $\psi \in \Gamma(L)$ such that $0<\psi(u)<\infty$. Thus $\Gamma(L)$ is separating.

Assume now that $\Gamma(L)$ is separating. Let $\left\{\phi_{\tau}\right\}$ be a maximal orthogonal system of positive elements of $\Gamma(L)$ and let $\phi=\sup \phi_{\tau}$. Then $0 \leqslant \phi, \phi \in \Gamma(L)$ and is strictly positive on its domain. This completes the proof of the theorem.

The following theorem will be used extensively.

Theorem 2.6. If $M$ is an order dense Riesz subspace of $L$, then $\Gamma(M)$ and $\Gamma(L)$ are isomorphic.

Proof. If $\phi \in \Gamma(L)$, then the restriction $\phi^{M}$ of $\phi$ to $M$ is obviously an element of $\Gamma(M)$. From the fact that $M$ is order dense in $L$ it follows immediately that the mapping $\phi \rightarrow \phi^{M}$ is one-to-one. Furthermore, it is linear and order preserving, and so in order to complete the proof we have to show that the mapping $\phi \rightarrow \phi^{M}$ is onto. To this end, let $0 \leqslant \phi^{\prime} \in \Gamma(M)$. I ts domain $D_{\phi^{\prime}}$ is an order dense ideal in $M$, and so $D_{\phi^{\prime}}$ is an order dense Riesz subspace of $L$. Now for every $0 \leqslant u \in L$ we set

$$
\tau_{\phi^{\prime}}(u)=\sup \left(\phi^{\prime}(v): 0 \leqslant v \in D_{\phi}^{\prime} \text { and } 0 \leqslant v \leqslant u\right) .
$$

It is obvious that $\tau_{\phi^{\prime}}(a u)=a \tau_{\phi^{\prime}}(u)$ for all $0 \leqslant a \in R$ and all $0 \leqslant u \in L$. Furthermore,

$$
\begin{aligned}
\tau_{\phi^{\prime}}\left(u_{1}\right)+\tau_{\phi^{\prime}}\left(u_{2}\right) & =\sup \left(\phi^{\prime}\left(v_{1}\right): 0 \leqslant v_{1} \leqslant u_{1}\right)+\sup \left(\phi^{\prime}\left(v_{2}\right): 0 \leqslant v_{2} \leqslant u_{2}\right) \\
& \geqslant \sup \left(\phi^{\prime}(v): 0 \leqslant v \leqslant u_{1}+u_{2} \text { and } v \in D_{\phi}\right)=\tau_{\phi^{\prime}}\left(u_{1}+u_{2}\right) .
\end{aligned}
$$


In order to prove the converse inequality, let $0 \leqslant v \leqslant u_{1}+u_{2}$ and $0 \leqslant v \in D_{\phi^{\prime}}$. Then

$$
v \leqslant u_{1}+u_{2}=\sup \left(v_{1}+v_{2}: 0<v_{1} \leqslant u_{1}, 0 \leqslant v_{2} \leqslant u_{2} \text { and } v_{1}, v_{2} \in D_{\phi^{\prime}}\right),
$$

and so

$$
v=\sup \left\{\inf \left(v, v_{1}+v_{2}\right): 0 \leqslant v_{1} \leqslant u_{1}, 0 \leqslant v_{2} \leqslant u_{2} \text { and } v_{1}, v_{2} \in D_{\phi^{\prime}}\right\} .
$$

But $\phi^{\prime}$ being normal implies that

$$
\begin{aligned}
\phi^{\prime}(v) & =\sup \phi\left(\inf \left(v, v_{1}+v_{2}\right)\right) \leqslant \sup \phi\left(v_{1}+v_{2}\right) \\
& =\sup \left(\phi\left(v_{1}\right)+\sup \phi\left(v_{2}\right)\right) \leqslant \tau_{\phi^{\prime}}\left(u_{1}\right)+\tau_{\phi^{\prime}}\left(u_{2}\right),
\end{aligned}
$$

and so $\tau_{\phi^{\prime}}\left(u_{1}+u_{2}\right) \leqslant \tau_{\phi^{\prime}}\left(u_{1}\right)+\tau_{\phi^{\prime}}\left(u_{2}\right)$. Hence

$$
\tau_{\phi^{\prime}}\left(u_{1}+u_{2}\right)=\tau_{\phi^{\prime}}\left(u_{1}\right)+\tau_{\phi^{\prime}}\left(u_{2}\right) \quad \text { for all } 0 \leqslant u_{1}, u_{2} \in L .
$$

Now we shall show that $0 \leqslant u_{\tau} \uparrow u$ implies that $\sup \tau_{\phi^{\prime}}\left(u_{\tau}\right)=\tau_{\phi^{\prime}}(u)$. Let $V=\{v: 0 \leqslant v \in L, v=u\}$, and $\operatorname{sosup}\left(\phi^{\prime}(v): v \in V\right)=\sup \tau_{\phi^{\prime}}\left(u_{\tau}\right)$. Now let $0 \leqslant v_{0} \leqslant u$ and $v \in D_{\phi^{\prime}}$. Then

$$
\phi\left(v_{0}\right)=\sup \left(\phi\left(\inf \left(v, v_{0}\right)\right): v \in V\right) \leqslant \sup \tau_{\phi^{\prime}}\left(u_{\tau}\right) .
$$

This shows that $\tau_{\phi^{\prime}}(u)=\sup \tau_{\phi}^{\prime}\left(u_{\tau}\right)$.

Finally, let $D=\left\{f: f \in L\right.$ and $\left.\tau_{\phi^{\prime}}(|f|)<\infty\right\}$. Then $D$ is an ideal in $L$ which contains the smallest ideal in $L$ containing $D_{\phi^{\prime}}$, and so is order dense in $L$. Furthermore, $\tau_{\phi^{\prime}}$ extends uniquely to a positive linear functional $\phi$ on $D$, and $0 \leqslant \phi \in \Gamma(L)$. Then it is obvious that the restriction $\phi^{M}$ of $\phi$ to $M$ equals $\phi^{\prime}$. This completes the proof.

We have seen that whenever $L$ is Dedekind complete and $\Gamma(L)$ is separating, then $L$ is an order dense ideal in $\Gamma^{2}(L)$. If $L$ is a band in $\Gamma^{2}(L)$, then $L=\Gamma^{2}(L)$. Any Riesz space satisfying this condition will be called perfect in the extended sense. A necessary condition for a Riesz space to be perfect in the extended sense is that it be Dedekind complete and universally complete. Analogously to Nakano's perfectness theorem (4, Theorem 28.4) the following theorem holds.

TheOREM 2.7. A Riesz space $L$ with the property that $\Gamma(L)$ is separating is perfect in the extended sense if and only if $0 \leqslant u_{\tau} \in L, u_{\tau} \uparrow$ and

$$
\sup _{\tau} \phi\left(u_{\tau}\right)<\infty
$$

for every $0 \leqslant \phi \in \Gamma(L)$ which belongs to some order dense ideal in $\Gamma(L)$ implies that $\sup u_{\tau}=u$ exists in $L$.

Proof. Let $L$ be perfect in the extended sense, and let $0 \leqslant u_{\tau} \uparrow$ with $\sup _{\tau} \phi\left(u_{\tau}\right)<\infty$ for all $0 \leqslant \phi \in I$, where $I$ is some order dense ideal in $\Gamma(L)$. Setting $p(\phi)=\sup \phi\left(u_{\tau}\right)$ for all $0 \leqslant \phi \in I$, then evidently we have $p(\phi) \leqslant 0$ and $p(\phi+\psi)=p(\phi)+p(\psi)$ for all $0 \leqslant \phi, \psi \in I$. Thus $p$ extends uniquely 
to a positive linear functional $p$ on $I$. In addition, since $0 \leqslant \phi_{\tau} \uparrow \phi$ on $I$ implies that

$$
p(\phi)=\sup \phi\left(u_{\tau}\right)=\sup _{\tau} \sup _{\lambda} \phi_{\lambda}\left(u_{\tau}\right)=\sup p\left(\phi_{\lambda}\right),
$$

it follows that $p \in I_{n} \sim$. Since $L$ is perfect in the extended sense, there exists an element $0 \leqslant u \in L$ such that $p(\phi)=\phi(u)$ for all $0 \leqslant \phi \in I$. Thus $\sup u_{\tau}=u$ in $L$.

From the hypothesis it follows that $L$ is Dedekind complete, and so $L$ is an order dense ideal in $\Gamma^{2}(L)$. Now let $0 \leqslant u_{\tau} \uparrow u^{\prime \prime}$ in $\Gamma^{2}(L), u_{\tau} \in L$ for all $\tau$. Then for all $0 \leqslant \phi \in D_{u^{\prime \prime}}$ we have $\sup \phi\left(u_{\tau}\right)<\infty$, and so $\sup u_{\tau}=u$ holds in $L$, i.e., $u=u^{\prime \prime}$ or equivalently $L=\Gamma^{2}(L)$.

Corollary 2.8. For any Archimedean Riesz space, the extended order dual $\Gamma(L)$ is perfect in the extended sense.

The proof is obvious.

3. Relations between $\Gamma(L), \mathfrak{D}(L)$, and $\mathfrak{D}\left(L_{n}^{\sim}\right)$. In $\S 1$, we showed that $L_{n} \sim$ can be embedded in a one-to-one way onto an ideal in $\Gamma(L)$. We shall identify $L_{n} \sim$ with this ideal.

THEOREM 3.1. Let $\Gamma(L)$ be separating; then, $L_{n} \sim$ is dense in $\Gamma(L)$ if and only if $L_{n} \sim$ is separating.

Proof. Suppose that $L_{n} \sim$ is separating. Let $\phi \in \Gamma(L)$. Then $\phi \in I_{n} \sim$ for some dense ideal $I \subset L$. By (2, Theorem 3.3), $L_{n} \sim$ is dense in $I_{n} \sim$. So $\phi$ is in the normal subspace generated by $L_{n} \sim$.

Suppose $L_{n} \sim$ is dense in $\Gamma(L)$. Given $u \in L, u \geqslant 0$, there is a $\phi$ in $\Gamma(L)$ such that $\phi(u)>0$. But there is a net $\left\{\phi_{\alpha}\right\}$ in $L_{n} \sim$ such that $\phi(u)=\sup \phi_{\alpha}(u)$ and hence a $\phi_{\alpha}$ in $L_{n}$ such that $\phi_{\alpha}(u)>0$.

Applying Theorem 2.6 to the above, we obtain immediately

THEOREM 3.2. If $L_{n} \sim$ is separating, $\Gamma\left(L_{n} \sim\right)$ is isomorphic with $\Gamma^{2}(L)$.

COROllary 3.3. If $L_{n} \sim$ is separating, $\Gamma(L)$ is a universal completion of $L_{n} \sim$.

Proof. $L_{n} \sim$ separating implies that $\Gamma\left(L_{n}^{\sim}\right)=\Gamma^{2}(L)$. So,

$$
\Gamma^{2}\left(L_{n}{ }^{\sim}\right)=\Gamma^{3}(L)=\Gamma(L)
$$

by Corollary 2.8. But, by Theorem $2.3, \Gamma^{2}\left(L_{n}\right)$ is a universal completion of $L_{n} \sim$.

Nakano has shown (8) that if $L$ is Dedekind complete, $\mathfrak{D}(L)$ and $\mathfrak{D}\left(L_{n} \sim\right)$ are isomorphic. Using this we obtain

Corollary 3.4. If $L_{n}^{\sim}$ is separating $\Gamma^{k}(L), \Gamma^{k}\left(L_{n}^{\sim}\right)$, $\mathfrak{D}\left(L^{\prime}\right)$, and $\mathfrak{D}\left(L_{n}^{\sim}\right)$, for each natural number $k$, are all isomorphic.

Proof. Nakano's result, in the light of Theorem 2.6, implies that $\Gamma^{2}\left(L_{n} \sim\right)$ 
and $\Gamma^{2}(L)$ are isomorphic and hence, by Corollary 2.8, $\Gamma(L)$ is isomorphic to $\Gamma\left(L_{n} \sim\right)$. Then, by Theorem $3.2, \Gamma(L) \cong \Gamma^{2}(L)$. The rest is easy.

Under the weaker assumption that $\Gamma(L)$ is separating, the space $L_{n} \sim$ may be trivial so we cannot hope to obtain all the isomorphisms. However, we do get the following theorem.

THEOREM 3.5. If $\Gamma(L)$ is separating, the spaces $\Gamma^{k}(L)$ and the space $\mathfrak{D}\left(L^{\prime}\right)$ are all isomorphic.

Proof. By Theorem 2.5, there is a dense ideal $I \subset L$ such that $I_{n} \sim$ is separating on $I$. Applying Corollary 3.4, $\Gamma(I)$ and $\Gamma^{2}(I)$ are isomorphic. Then, by Theorem 2.6, $\Gamma(L)$ and $\Gamma^{2}(L)$ are isomorphic. The rest follows easily.

5. Some examples. We mention first, by way of examples, that $\Gamma(L)$ may not be separating on $L$ whether $L$ is complete or not. If $C=C([0,1])$ (the continuous functions on the closed interval $[0,1]$, then $\Gamma(C)=0$. If $C^{\wedge}$ is the cut completion of $C$, then $\Gamma\left(C^{\wedge}\right)=0$. The first result is obtained by characterizing the dense ideals of $C[0,1]$ and showing that they have no continuous linear functionals. The second is merely the isomorphism obtained in a previous section.

Two particular examples influenced the development of the space $\Gamma(L)$. The first is $\mathfrak{M}$, the equivalence classes of measurable functions defined on the closed interval $[0,1]$. M shares with $C([0,1])$ the property that it has no non-trivial continuous linear functionals. Yet, ideals in $\mathfrak{M}$ are rich in linear functionals whereas this is not true in $C([0,1])$. Might $\mathfrak{M}$ then possess continuous linear functionals in the "unbounded" sense?

Let $L^{\infty}=L^{\infty}([0,1])$. The universal completion of $L^{\infty}$ is clearly $\mathfrak{M}$. So, $\Gamma\left(L^{\infty}\right)=\mathfrak{M}$. But, $L^{\infty}$ is a dense ideal in $\mathfrak{M}$; hence $\Gamma(\mathfrak{M})=\Gamma\left(L^{\infty}\right)$. So, not only does $\mathfrak{M}$ have a non-trivial unbounded dual $\Gamma(\mathfrak{M})$ but also $\mathfrak{M}=\Gamma(\mathfrak{M})$.

Secondly, in $(\mathbf{1}, \mathbf{2}, \mathbf{4}) \mathrm{S}$. Kaplan studies the space $C(X)$ (continuous realvalued functions on the compact Hausdorff space $X$ ), through the embedding of $C(X)$ in its second dual space $M$. One of the basic results to this study is the existence of a closed ideal $M_{0}$ in $M$ which is isomorphic and isometric to the bounded functions on $X$. In (1), there arise questions concerning the space $S$ of differences of lower semicontinuous elements in $M$. One of the difficulties hinges on the fact that a bounded function may be the difference of two (unbounded) lower semicontinuous functions and yet cannot be expressed as the difference of two bounded lower semicontinuous functions. How, then, might one generate a larger second dual space $M$ in which one can represent unbounded functions on $\mathrm{X}$ ?

Let $L=(C(X))^{\sim}$ (the bounded linear functionals on $C(X)$ ). Then $M=L^{\sim}=L_{n}^{\sim}$ since $L$ is an abstract $L$-space. Moreover, $L_{n}^{\sim}$ is separating on $L$. So, let $M^{\wedge}=\Gamma(L)$. Then, $M^{\wedge}$ contains $M$ as a dense ideal. Moreover, if we let $M_{0}^{\wedge}$ be the order closure of the ideal $M_{0}$ in $M^{\wedge}, M_{0}^{\wedge}$ turns out to be isomorphic to the space of all real-valued functions on $X$. 


\section{References}

1. S. Kaplan, The second dual of the space of continuous functions II, Trans. Amer. Math. Soc., 93 (1959), 329-350.

2. - The second dual of the space of continuous functions III, Trans. Amer. Math. Soc., 101 (1961), 34-51.

3. - The second dual of the space of continuous functions $I V$, Trans. Amer. Math. Soc., 113 (1964), 512-546.

4. W. A. J. Luxemburg and A. C. Zaanen, Notes on Banach functions spaces VIII, Nederl. Akad. Wetensch. Proc. Ser. A, 67, 1 (1964), 104-119.

5. - Notes on Banach functions spaces IX, Nederl. Akad. Wetensch. Proc. Ser. A, 6, 1 (1964), 360-376.

6. - Notes on Banach functions spaces $X$, Nederl. Akad. Wetensch. Proc. Ser. A, 67, 1 (1964), 493-506.

7. - Notes on Banach functions spaces XVI, Nederl. Akad. Wetensch. Proc. Ser. A, 68, 1 (1965), 646-667.

8. H. Nakano, Modern spectral theory (Tokyo, 1950).

California Institute of Technology

and Michigan State University 